\title{
A Co-simulation Framework for Assessing the Interaction between Heat Pumps and the Low Voltage Grid on a District Scale
}

\author{
Jalomi Maayan Tardif ${ }^{1,2,3}$, Francisco Diaz-Gonzalez ${ }^{2}$, Martin Kegel ${ }^{3}$, Alaia Sola ${ }^{1}$, Jaume Salom ${ }^{1}$ \\ ${ }^{1}$ Catalonia Institute for Energy Research - IREC, Sant Adrià de Besòs (Barcelona), Spain \\ ${ }^{2}$ Universitat Politècnica de Catalunya, Barcelona, Spain \\ ${ }^{3}$ Natural Resources Canada - CanmetENERGY, Varennes, Canada
}

\begin{abstract}
The traditional unidirectional-flow electricity grid is evolving and integrating active power electronics, controllable loads, and Distributed Energy Resources (DERs). The design and validation of innovative electric networks is challenging and since experimental data is not easily obtained on such large scales, simulation-based approaches are adopted to support their development. The simulation and validation of the grid at a system level requires high resolution models of multiple domains involving the end user, the electric grid, and the interaction between them, and a co-simulation approach is adopted in order to encompass all elements and take advantage of the many specialized tools that address each of them separately. This paper will present a co-simulation framework that uses the Functional Mock-up Interface (FMI) standard in order to combine TRNSYS for the building domain and the Python package PandaPower for the electric grid domain.
\end{abstract}

\section{Introduction}

Reducing Green House Gas (GHG) emissions while preserving the quality of the energy supply is a major challenge in the energy sector today. As buildings represent approximately 33\% of the global primary energy use, coordinating the energy supply and demand in this sector could have a significant impact on GHG reduction as well as potentially provide flexibility to the energy system (European Commission (EC), 2016; Jensen et al., 2017; Péan, Salom, and Ortiz, 2017). Two main strategies are adopted when tackling energy consumption in the residential sector; the first involves reducing the energy consumption by means of highly efficient materials and technologies, and the second aims at increasing the generation of electricity from renewable sources for self-consumption. Heat Pumps (HPs) have been found to support both strategies, as they provide heat with less energy input as compared to fossil fuel fired boilers, and they provide a link between the heat and the electricity domains, thereby providing an opportunity to use renewable energies to meet the Heating, Ventilation and Air-Conditioning (HVAC) loads. Moreover, HVAC and Domestic Hot Water (DHW) loads can partly be shifted in time and as such HPs can help decouple the electricity consumption required for these loads from the heating/cooling demand of the building, thereby providing potential flexibility and supporting higher penetration rates of DERs in the electric grid. However, the widespread use of HPs could possibly lead to grid congestions, particularly at the Low Voltage (LV) level since the distribution equipment may not be adequately dimensioned for residences with an electric-based heating system with higher consumption peaks. As long as the HP and DER penetration rates are low, the conventional distribution network can still function without important reinforcement investments. However, as the penetration rate increases, there is a potential risk of voltage deviations from the permitted voltage range $( \pm 10 \%$ of the rated voltage according to EN 50160), as well as local overloads of the grid equipment, especially of the power cables and transformers (Akmal et al., 2014; NavarroEspinosa and Mancarella, 2014). To allow a further electrification of the residential heat sector while guaranteeing a stable operation of the grid, various solutions have been offered:

1. Investment in additional LV equipment, such as new transformers and cables. Such investments are considered cost-intensive, especially in the LV grid which involves underground cables.

2. Investment in automation devices that allows to use the currently installed equipment more efficiently by means of controllable loads and power electronics.

3. Investment in DERs for self-consumption and/or grid generation support.

The coupling and integration of existing energy systems with advanced new automation devices and renewable DERs is expected to provide an efficient and stable system, with less GHG emissions. However, the control strategy, and related cost as compared to investment in additional traditional grid equipment, have yet to be clearly identified. For instance, the concept of the cooperation of a pool of HPs that link the electrical and thermal domains in an optimal synergy, could increase energy efficiency and energy savings. However, the scale of the HP pool that would provide a valuable flexibility to the grid, and the control strategy that would best serve all involved stakeholders, is currently in the research phase. The modelling and simulation of such multi-domain systems is the key to understand their complexity, strength, and weakness, both in the design phase and during operation. Unfortunately, traditional power and energy related simulation tools are typically restricted to specific domains, and therefore the assessment of multidomain solutions, where the interaction between the domains is of critical importance, is infeasible (Widl et al., 2015). 
The following paper presents a co-simulation framework that allows to assess the power-flow in a LV feeder with high resolution stochastic residential electrical-load profiles. The co-simulation platform allows to send grid-to-building feedback, however this paper will focus on the impact of the HPs in the current passive grid, in order to assess the risk at the LV level to begin with. Four scenarios with increasing penetration rates of HPs will be presented in order to assess their impact on the grid, and if they create any stress on the grid equipment that would require any of the interventions mentioned. Additionally, as urban and rural grids characteristics differ, a sensitivity analysis is performed by simulating the scenario with a HP penetration rate of $100 \%$ in four grids that vary in terms of the number of connected customers and the distance between the delivery points. The impact results are given in terms of the voltage levels and the transformer loading. While the focus of the paper is on the Spanish housing sector the strategy outlined was performed in a Swedish scenario as well (Maayan Tardif, Diaz-Gonzalez, and Salom, 2018), and can be replicated in other regions seeking information on the grid impact of heat pumps, which can vary widely, depending on the climate and the dominating conventional HVAC technology. The assessment is especially interesting for Canada having set Greenhouse Emission reduction targets and in order to do so, have set aspirational goals that all space and water heating systems must have seasonal efficiencies greater than $100 \%$ by 2035 (Energy and Mines Ministers' Conference, 2017). As such a switch from oil and natural gas heating systems to electrically driven systems are foreseen, which can have an impact on both the urban and rural grid.

\section{Co-simulation Framework}

The following will briefly describe the main components of the framework which is graphically represented in Figure 1. The co-simulation framework consists of a Python script "Master" which at each time-step communicates via the Functional Mock-up Interface (FMI) standards with multiple individual buildings which are modelled in the specialized simulation software TRNSYS. The total electric demand calculated by TRNSYS for each building is sent back to the "Master" which then passes the information onto the electric grid modelled in PandaPower for a power-flow analysis.

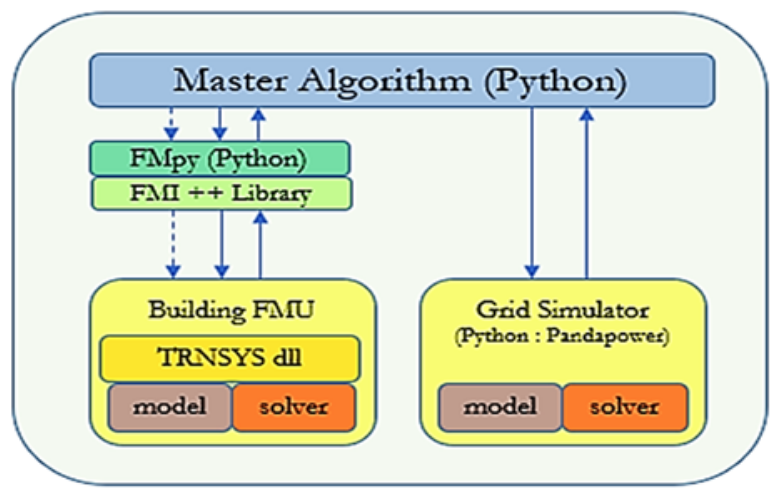

Figure 1: Graphical representation of the co-simulation framework

\section{Functional Mock-up Interface (FMI)}

FMI (MODELISAR consortium, 2014) is a general standard which is tool-independent. The FMI specifications define the interfaces that enable various simulation tools to interoperate, using C-language to define the stages of instantiation and initialization, as well as the configuration that allows access, modification and manipulation (Wang, Siebers, and Robinson, 2017). A simulation model that complies with the FMI standard is wrapped in a Functional Mock-up Unit (FMU). The FMI specification defines two specific types of interfaces (Eder et al., 2016):

- FMI for Co-Simulation (CS) is used in the case of a stand-alone black-box model. Data exchange is limited to discrete communication points, between which the system is solved by the FMU's internal solver.

- FMI for Model Exchange (ME) is used to access a model, however the master algorithm supplies the solver. In such case the simulation can be timecontinuous.

The FMU is distributed as a zip-folder that contains three main elements; an XML-based model description file, a resources folder with extra data files needed for the model, and a binaries folder with the source code implementing the actual interface in $\mathrm{C}$ code. Which type of FMU is used depends on the tools that are being coordinated and the co-simulation environment.

\section{TRNSYS}

The simulation tool used is TRNSYS v.17 (Solar Energy Laboratory Univ. of Wisconsin-Madison et al., 2017). TRNSYS is a dynamic simulation tool focused on modelling buildings and their related systems. In TRNSYS, a model is developed within a graphical interface environment with pre-developed components and algorithms which model the behaviour of common building systems. TRNSYS also allows the user to develop custom components, which can also be used to establish links to other software important in a co-simulation environment. The model is saved in a "deck" file (.dck), which stores the components and connections between them. The model is executed by TRNExe, and an algebraic and differential equation solver iteratively computes the system state and seeks convergence at each time step.

\section{Python}

Python is an object-oriented, high-level general-purpose programming language that can be applied to many different classes of problems. It is an open source development project, with an active community of contributors which offer multiple free Python based packages, of which the following were used in this co-simulation framework:

- FMPy - A free Python library used to access FMUs. It supports FMI 1.0 and 2.0 for both Co-Simulation and Model Exchange, compiling C-code FMUs (Dassault Systèmes, 2018). 
- PandaPower - combines the data analysis library pandas and the power-flow solver PyPower to create networks to be analysed and optimized (Thurner and Scheidler, 2017).

The PandaPower tool was validated by running a static powerflow of the European CIGRE LV benchmark grid (CIGRE, 2013) and comparing the voltage and current results. The resulting PandaPower node voltage in the residential section of the grid matches very well with the benchmark results, with an average difference of $0.95 \%$, however a gap was found in the line current results, with an average difference of $14.91 \%$. The minor discrepancy among the CIGRE benchmark results and the developed model occurs since the CIGRE benchmark deals with unbalanced systems and the simulated model considers a balanced one, leading to impedance modifications. Yet, assuming a balanced three-phase system is valid and has been used in other studies of the LV grid by researchers and major DSO companies (Mehmedalic, Rasmussen, and Harbo, 2013).

\section{Case Study}

The case study represents a south European climate and is based in the area of Barcelona (Spain). A sequence of five days is simulated with a time step of three minutes, where the first two days serve the purpose of initializing the state of variables and are discarded from the analysis which focuses on the three subsequent days. According to the Joint Research Center (JRC) of the European Commission, the main load in an average European residence is heating, however in the Mediterranean climate of Spain the heating and cooling loads are relatively balanced (Zangheri et al., 2014). Therefore, this study tested two sequences of five critical days with extreme cold and hot weather, representing worst-case scenarios in terms of heating and cooling loads, respectively.

\section{Building Model}

The following will describe the characteristics of the residential model that was previously developed in TRNSYS and validated (Ortiz et al., 2016). The building has a floor area of nearly $108.5 \mathrm{~m}^{2}$ on a single level and represents a common single-family house building typology in this region. The residence is an apartment that is part of a multi-unit residential building built to meet the Spanish codes from the 1980s and the main characteristics are found in Table 1.

Table 1: Summary of building characteristics

\begin{tabular}{|c|c|c|}
\hline Parameter & Unit & Value \\
\hline Floor area & $\mathrm{m}^{2}$ & 108.5 \\
\hline Roof U-value & $\mathrm{W} / \mathrm{m}^{2} \mathrm{~K}$ & 0.546 \\
\hline Wall U-value & $\mathrm{W} / \mathrm{m}^{2} \mathrm{~K}$ & 0.6 \\
\hline Window U-value & $\mathrm{W} / \mathrm{m}^{2} \mathrm{~K}$ & 2.5 to 5.7 \\
\hline Infiltration $\mathrm{n} 50$ & $1 / \mathrm{h}$ & 3.0 \\
\hline
\end{tabular}

\section{HVAC}

The base case heating system consists of a conventional natural gas boiler which feeds hydronic radiators, which is the dominant technology used in this region (Boneta and Sebi, 2012). This system is replaced by a Variable Speed Air-to-Water Heat Pump (VS AWHP) which aims at maintaining the set-point temperature and comfort of the occupants, regardless of time-of-use electricity tariffs or other more complex control strategies. The choice of an air-to-water system in the Spanish case study is explained in Tejero et al. (2018) as the only realistic choice for a hydronic system that is to supply DHW as well. The VS AWHP was developed by Toffanin (2018) and is based on available manufacturer data. The performance of the HP follows two distinct operation modes. When the conditions impose a Part-Load Ratio (PLR) is below the minimum operational limit of the HP, the Part-Load Factor (PLF) equation developed by Fuentes et al. (2016) is used, while when above that limit the PLF-PLR curve is based on available manufacturer part-load data using a quadratic fit method.

\section{Occupancy and Appliances}

Residential energy consumption profiles are difficult to predict for several reasons: occupant behaviour can vary widely, privacy issues limit the access to individual household energy data, and usually the detailed metering of end-uses consumption are costly. The importance of the demand side modelling is particularly high when evaluating the peak consumption of an aggregated building cluster, upon which relies the design of the LV grid. The high importance of load diversity is highlighted in Schwalbe et al. (2017) where the load profiles used in one grid model had a high coincidence factor and consequently the base case rural Austrian grid modelled suffered from a voltage level violation, even before adding HPs.

As such, it was chosen to use 25 stochastic profiles developed by Ortiz et al. (2014) which represent homes with four occupancy levels and three appliance stock levels. The proportions of residences with 1, 2, 3 or 4 occupants on a LV feeder is weighted according to the statistical data (European Commission, 2014) provided in Table 2 .

Table 2: Occupancy levels of dwellings on the feeder

\begin{tabular}{|c|c|c|c|c|}
\hline Number of occupants & 1 & 2 & 3 & 4 \\
\hline $\begin{array}{c}\text { National percentage of } \\
\text { residences }\end{array}$ & 24.6 & 30.6 & 21.1 & 17.8 \\
\hline $\begin{array}{c}\text { Number of residences on } \\
\text { base case feeder }\end{array}$ & 6 & 8 & 6 & 5 \\
\hline
\end{tabular}

The peak power of the appliances of the 25 single household models are within a range of $2.6-6.8 \mathrm{~kW}$, and the addition of a HP increases the household loads, to a range of 4.0-7.1 kW, which is within the range found in European networks (Prettico et al., 2016; Salom et al., 2014). A power factor of 0.95 is used, as prescribed by CIGRE and also found in other studies such as Flammini and Prettico (2017).

The time-step resolution is also an important factor in the interaction between a residence and the LV grid. A study by (Salom et al., 2015) presents a comparison between the peak load of a dwelling simulated with hourly data and the peaks obtained with higher resolution data of 12-minute, 5-minute, and 1-minute time-steps, and 
concludes that the hourly data tends to smoothen the power demand profiles there and that there can be a significant difference of $17 \%, 73 \%$, and $69 \%$, respectively, for the decreased time-steps. Therefore this study used a 3 minutes time-step which could be considered adequate for capturing the magnitude of the "peaks" and "valleys" of the individual electricity demand profiles, which is of special concern for network operators (Marzal-Pomianowska et al., 2016).

\section{Electric Grid Model}

Rather than evaluating entire networks with many feeders, a LV feeder was evaluated individually. This choice follows previous research that has found that most challenges experienced in LV networks relate to the individual feeders due to their radial topology (Mehmedalic et al., 2013). The CIGRE LV grid benchmark network is modelled in PandaPower and consists of an MV/LV transformer with a capacity of $20 / 0.4 \mathrm{kV}, 500 \mathrm{kVA}$, which is followed by a busbar from which branch two feeders. A graphical representation is presented in Figure 2, where the base-case feeder supplies 25 houses distributed into 5 clusters of buildings ( $R 11$ and $R 15-R 18)$. The load $R 1$ represent the second feeder, so that the transformer serves two identical feeders for a total of 50 consumers, which is in accordance with the value found in a survey of 79 of the 190 largest DSOs in Europe (Mateo et al., 2018).

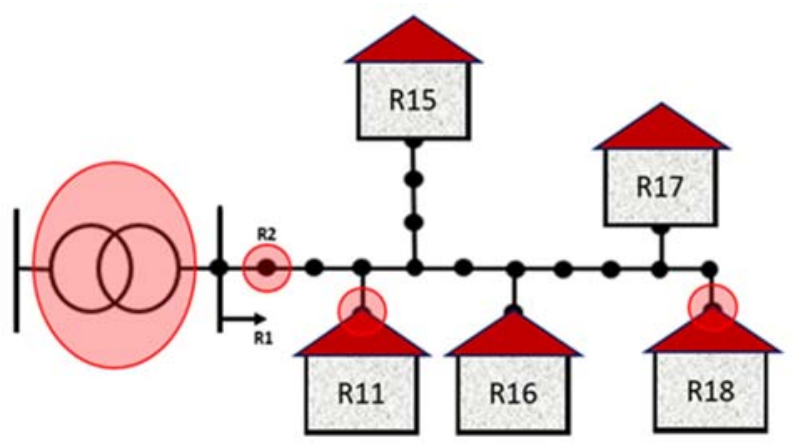

Figure 2: Simplified representation of the LV grid. In red circles are the transformer and the nodes at the feeder extremities, which are identified as of interest for the study.

The levels of occupancy of the households is faithful to the typical distribution in the building stocks of Spain (previously given in Table 2) and the profiles are randomly distributed amongst the dwellings in the five building clusters as described in Table 3.

Table 3: Base-case number of dwellings in each cluster

\begin{tabular}{|c|c|c|c|c|c|}
\hline Dwelling cluster & R11 & R15 & R16 & R17 & R18 \\
\hline Number of dwellings & 1 & 11 & 4 & 3 & 6 \\
\hline
\end{tabular}

In order to evaluate the impact of HPs on the LV grid, four HP penetration rates are evaluated; i) $H P \_0$, ii) $H P \_30$, iii) $H P \_60$, and iv) HP_100, which represent scenarios with $0 \%, 30 \%, 60 \%$, and $100 \%$ HP penetration in the grid, respectively. The residences where the boiler is replaced by a HP are randomly chosen. Typically, rural and urban grids are characterized differently in terms of the number of customers, the load magnitude, and the distance between the customers. Therefore, an additional sensitivity analysis will evaluate four scenarios with different energy densities in terms of load per feeder length; i) Rural Base-Case $\left(R \_B C\right)$, ii) Rural Worst-Case $\left(R \_W C\right)$, iii) Urban Base-Case $\left(U \_B C\right)$, iv) Urban WorstCase (U_WC). In all scenarios the topology of the grid is maintained as in the CIGRE benchmark, however the length of the cable between delivery points is altered such that the LV circuit length per LV customer are similar to values provided in the "Distribution system operators observatory" report by (Prettico et al., 2016). In $R \_W C$ the length between delivery points is doubled, while in $U_{-} W C$ the number of customers is doubled, for a total of 100 customers for the MV/LV transformer which concords with grid models described in Wilkening et al. (2016), Dickert et al. (2013), and Mateo et al. (2018). In this analysis all cases have a HP penetration rate of $100 \%$. The properties of each of these grids are found in Table 4 . The total load at the transformer "S $\mathrm{S}_{\max }$ " corresponds to a case where all residences would experience a peak at the same time (coincidence factor $=1$ ), and " $\mathrm{S}_{\mathrm{o}}$ " is the actual peak load which occurs in the presented case-study simulated with individual stochastic profiles. Both values correspond only to the appliance and lighting loads, which represent to the total electric load in the base case where the HVAC is supplied by a natural gas boiler. The values for $R \_B C, R \_W C$, and $U \_B C$ are the same because they have the same number of customers.

Table 4: General feeder characteristics

\begin{tabular}{|c|c|c|c|c|}
\hline Grid Type & R_BC & R_WC & U_BC & U_WC \\
\hline Feeder length [m] & 570 & 990 & 106 & 106 \\
\hline Customer / feeder & 25 & 25 & 25 & 50 \\
\hline meter / customer & 22.8 & 39.6 & 4.24 & 2.12 \\
\hline $\mathrm{S}_{\max }[\mathrm{kVA}]$ & 117.3 & 117.3 & 117.3 & 234.5 \\
\hline $\mathrm{S}_{\mathrm{o}}[\mathrm{kVA}]$ & 33.6 & 33.6 & 33.6 & 67.24 \\
\hline $\mathrm{kVA} /$ meter & 0.06 & 0.03 & 0.32 & 0.63 \\
\hline
\end{tabular}

\section{Key Performance Indicators}

A power flow analysis of the LV grid will allow to evaluate if the voltage deviation at the first node $(R 2)$ and last nodes (R18) on the feeder remain within an allowable range of $\pm 7 \%$ of the nominal voltage, which is more conservative than the $\pm 10 \%$ standard but can be used by DSOs in the design of a grid (Müller et al., 2015; Rodriguez-Calvo, Cossent, and Frías, 2017). The voltage levels will be expressed in terms of "per unit" (p.u), where the nominal voltage of $230 \mathrm{~V}$ is 1 p.u. and the lower limit is 0.93 p.u. Additionally, the loading of the transformer in terms of percentage of its capacity is evaluated, where it is considered to be overloaded when the load surpasses $80 \%$ of the transformer rated capacity. Moreover, the risk of accelerated aging of the transformer is evaluated with a Utilization Index (UI) which is the ratio of time in overload per day.

\section{Results and Discussion}

In this section will first be presented the results of the power-flow in the case of increasing HP penetration rates, followed by the analysis of the sensitivity to the grid configuration and load density. Then will be discussed 
some of the technical computational aspects of the cosimulation platform.

\section{HP penetration rates}

The following results will focus on the Rural Base-Case $\left(R \_B C\right)$ as it resembles most the configuration of the CIGRE benchmark LV grid. The stochasticity of the profiles plays an important role in the final peak power of the dwelling clusters, as, for example in $R \_B C$, the single residence (R11) has the highest peak per customer of $5.65 \mathrm{kVA}$, and the cluster of 11 residences (R15) has the lowest peak per customer of $2.66 \mathrm{kVA}$. The peak per customer is an important factor in the design of the LV grid. Common methods to size distribution grids are the Velander formula and methods based on simultaneity and coincidence factors. These methods are considered reliable when the type of individual loads is relatively homogeneous, (e.g. all residential), and the number of residences is large enough (above 200 connections), however there is some doubt about the reliability of this method for feeders serving smaller cluster, such as 20-60 residences. When comparing the resulting aggregated loads of the Spanish (SPN) case-study with common grid design methods such as the Velander method (VL) or the Simultaneity Factor (SF) Spanish regulations method (Sartori et al. 2014), the general trend is similar (Figure $3)$.

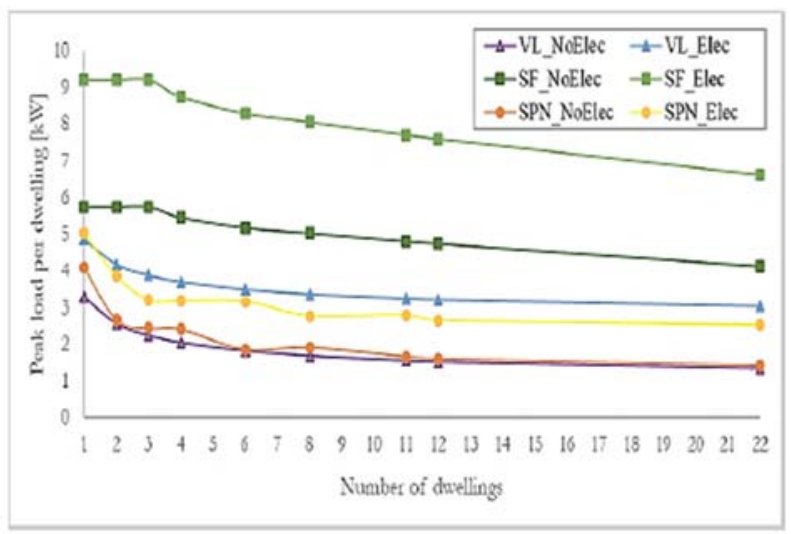

Figure 3: Peak power per customer with increasing dwelling aggregation levels

It can be seen that although the coefficients used in the Velander's formula are general values for domestic buildings with and without electric heating, and not specifics for Spain, the shape of the curve for the basecase residence with no electric heating (SPN_NoElec) follows closely the trend of the Velander method with no electricity (VL_NoElec) and that when the electric heat pumps are integrated (SPN_Elec), then the general trend is similar to the Velander method curve for residences with electric heating (VL_Elec). It can also be noted that, similarly to the case presented by Satori (2014), the Spanish Simultaneity Factor generally seem to be more conservative and estimates higher loads.

The voltage deviation of the first node $R 2$ ("V2") and last node $R 18$ ("V18") on the feeder is shown in Figure 4.

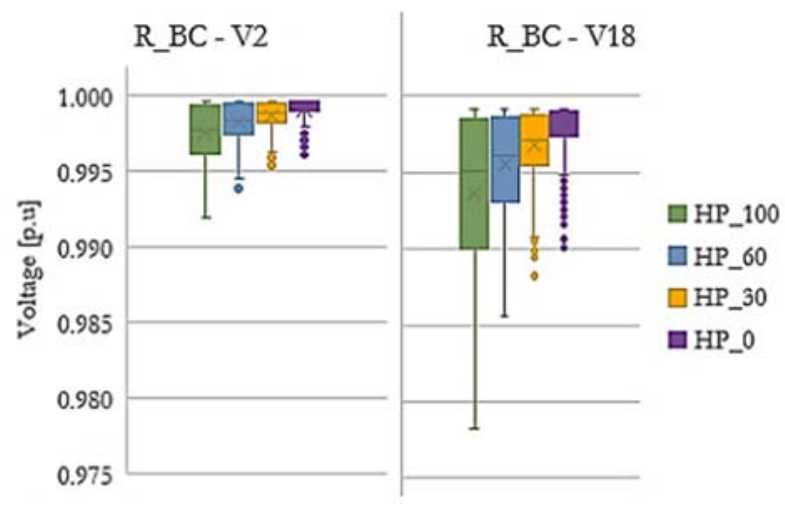

Figure 4: Voltage levels at the first feeder node (V2) and last feeder node (V18) for different HP penetration rates in the Rural Base Case (R_BC)

It can be seen that the additional HPs cause the electrical load to increase and therefore the voltage levels in both $V 2$ and V18 tend to drop. It can also be noted that the difference between the maximum and minimum voltage levels increases as the HP level increases, however the minimum value reached is 0.979 in the scenario with a HP penetration of $100 \%$, and remains in all scenarios above the lower limit of 0.93 p.u.

The transformer loading in closely related to the electrical load in the grid which increases as the HP penetration rate increases. In Figure 5 can be seen that in a purely residential system with only conventional natural gas boilers (HP_O) the only load on the LV feeder is the electric appliances, reaching a peak load which corresponds to $12 \%$ of the transformer capacity. As the HP penetration rate increases, the peak load increase by $14 \%, 19 \%$ and $25 \%$ for the HP_30, HP_60, and HP_100 scenarios, respectively (percent difference is $17 \%, 45 \%$ and $70 \%$ ).

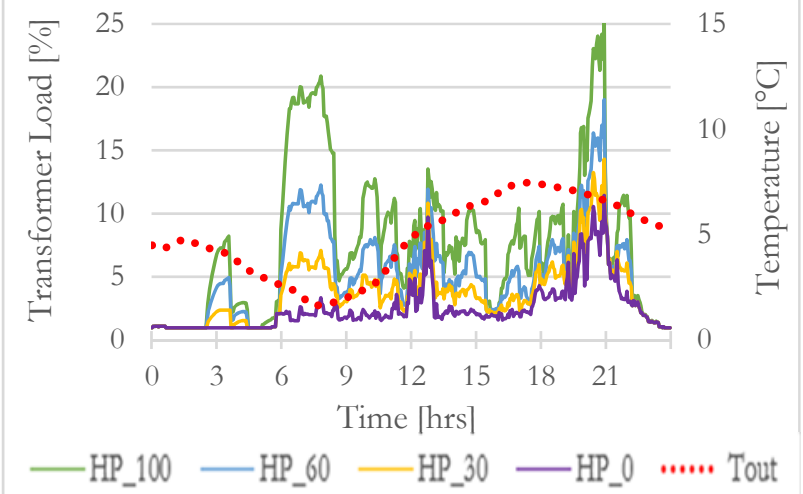

Figure 5: Spanish $R \_B C$ transformer loading

As can be seen from the daily transformer loading profiles, the peak loading does not necessarily correspond with the outdoor temperature, indicating that the HP does not always dictate the demand profile. Although the HP causes an additional peak in the morning between 6:00-9:00 when the temperatures are low, over the period analysed, the daily electrical load of the appliances and lighting was higher than the heating load between $50 \%$ to $75 \%$ of the day. In all cases the transformer is far from being overloaded, which means that the increasing 
integration of HPs does not critically accelerate the aging of the transformer and the utilization index in this case is zero.

\section{Load Density}

The apparent power load of each dwelling cluster in winter and summer can be seen in Figure 6 and Figure 7, respectively. For example, S11_BC and S11_WC represents the apparent power in the first cluster R11 in the Urban Base Case (U_BC) case and Urban Worst Case (U_WC), respectively. It can be seen that the heating load is slightly higher than the cooling load, with a peak in the largest cluster R15 (S15_WC) of $52.6 \mathrm{kVA}$ and $42.3 \mathrm{kVA}$, in winter and summer, respectively, and therefore all the following description of results will refer to the winter scenario.

As expected, the lowest voltage level is experienced at R18 in the rural worst-case scenario with the longest distance between delivery points and the lowest load density. When comparing R_BC and R_WC it can be seen that doubling the cable length caused the voltage drop to increase by 0.014 p.u. $(3.22 \mathrm{~V})$. However, even in this case the minimum value reached is 0.964 (Figure 8), which is above the lower limit of 0.93 p.u.

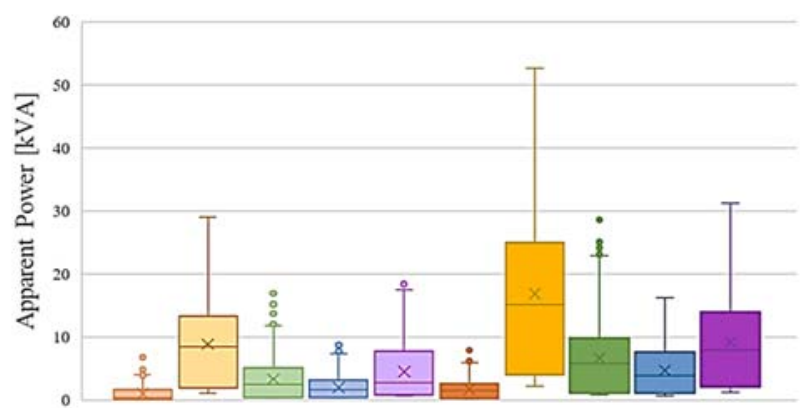

$\square \mathrm{S} 11 \_\mathrm{BC} \square \mathrm{S} 15 \_\mathrm{BC} \square \mathrm{S} 16 \_\mathrm{BC} \square \mathrm{S} 17 \_\mathrm{BC} \square \mathrm{S} 18 \_\mathrm{BC}$ S11_WC $\square$ S15_WC $\square$ S16 WC $\square$ S17_WC $\square$ S18_WC

Figure 6: Winter load of each dwelling cluster for U_BC and $U_{-} W C$

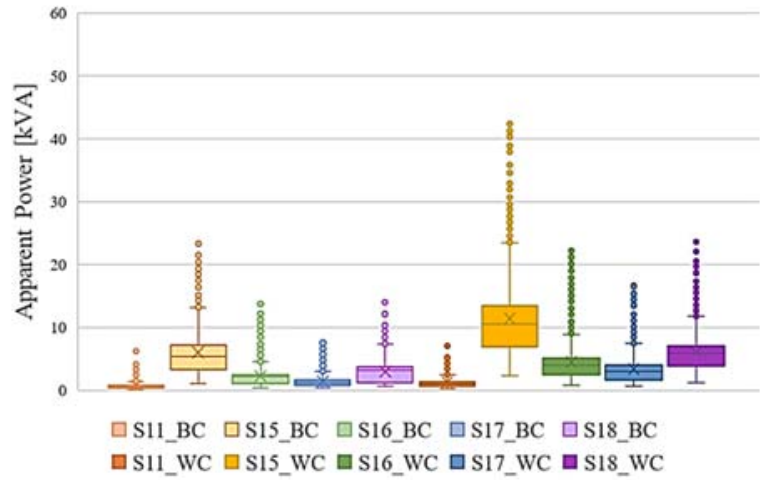

Figure 7: Summer load of each dwelling cluster for $U \_B C$ and $U \_W C$

The loading of the transformer in $R \_B C, R \_W C$, and $U \_B C$ is very similar as they have the same number of residences, and apparently increasing or decreasing the cable length does not have much effect. However, doubling the number of residences in the U_WC scenario proportionally increased the peak loading on the transformer, from $24.91 \%$ in the U_BC to $50.21 \%$ of the transformer capacity.

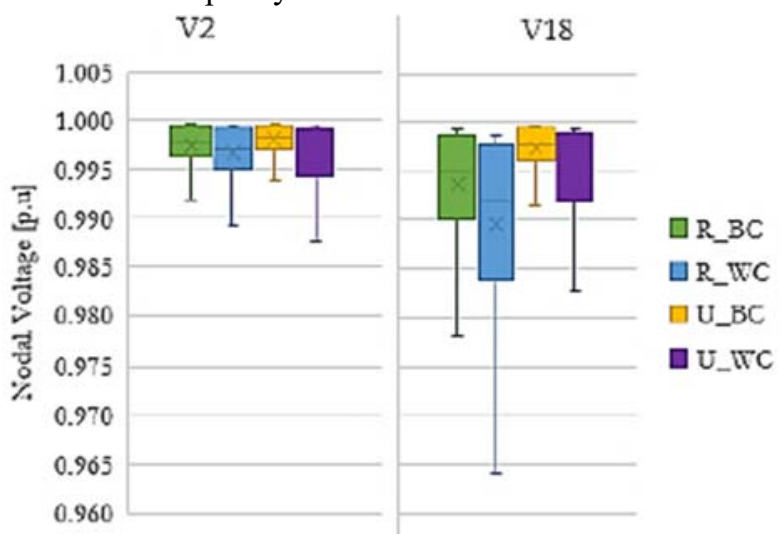

Figure 8: Voltage levels at the first feeder node (V2) and last feeder node (V18) for different grid configurations with a HP penetration rate of $100 \%$.

Technical Evaluation of the Co-Simulation Platform

The interaction between the grid model and the building model with high resolution demand profiles is possible with this co-simulation platform. The running time of the simulation from the starting initialization in the command prompt window, to the final graphics and written csv result files, when performed on a 2 cores laptop with a base frequency of $1.7 \mathrm{GHz}$, took on average 68 minutes, for a 5-day simulation length using a time-step resolution of 3 minutes. Comparing this execution time to standalone TRNSYS and Python models is not straightforward, however it can be approximated by summing the simulation time for 25 individual TRNSYS models and the time for the Python model to read the resulting aggregated loads a perform a power flow for each timestep. The PandaPower power-flow of the 5 days takes approximately 1.5 times longer when it extracts the power load from multiple FMUs rather than from a single file. From the TRNSYS graphical user interface it is possible to run simultaneously multiple separate models containing a type-56 (used for multi-zone building modelling), however it increases the simulation time for each single model. For example, a standalone simulation of the TRNSYS model of the Spanish residence took on average 1.9 minutes, however when manually starting 6 simulation models one after the other, so that they run at the same time, increased the simulation time of each separate model, for a duration between 2.18-3.78 minutes, with an average of 2.88 minutes. The total time for all 6 simulations to be completed was between 5.18-6.02 minutes, with an average of 5.46. Therefore, it can be assumed that manually initializing 25 separate models consecutively and post-processing the results into a single file that will be used for the power-flow could take at least to 15 minutes. As such, it can be estimated that the total simulation time of the co-simulation platform is roughly similar to what would be required for simulating the system with the same tools separately. Consequently, although this is only a rough estimate of computational time, it can be said that the co-simulation platform not 
only adds the possibility for the building and grid models to send feedback to each other during the simulation process, but also does not compromise the calculation time. As this research focused on a LV benchmark, the platform has yet to be tested for larger building clusters and the simulation time or processing power required is unknown, although not very larger clusters than the ones tested here are expected for LV networks

\section{Conclusions and Future Work}

When designing innovative grids there are many stakeholders that need to be considered:

- Households are concerned about the cost of energy, which is determined by the effective net energy balance of all energy sources purchased or locally produced, and possible discomfort,

- DSOs are concerned with the voltage quality and the transformer loads, and the cost of the distribution equipment that maintains theses aspect within acceptable ranges.

- Public authorities are concerned about the net energy balance and the resulting equivalent GHG emissions.

In order to properly address the interests of each and all stakeholders, a simulation platform needs to be able to model each component of the system and the interaction between them. The goal of this work was to use cosimulation to take advantage of specialized simulation tools for cross-domain collaboration. The use of FMI successfully linked between the building and the grid models and enables pre-feasibility studies that allow highresolution analysis of a multitude of aspects of the grid.

The case study of a benchmark European grid in a Mediterranean climate demonstrated the capacity of the platform to evaluate different scenarios and future work could test colder climates where the heating load is higher and therefore there is a higher risk of stressing the grid equipment. Moreover, although the work presented in this paper only evaluates a passive system, the response of TRNSYS to random control signals sent from the master algorithm has been tested successfully, and therefore this platform could support testing various grid-driven strategies involving the control of HPs on an aggregated level. This platform could also be used to assess the power flow in a grid with DERs and test control algorithms that would provide stability and flexibility to the power system.

\section{Acknowledgement}

This research was conducted in a framework of collaboration under the supervision of Jaume Salom from IREC who conducted this research under the GEIDI project (ref. TIN2016-78473-631 C3-3-R) financed by the Ministry of Economy and Competitiveness of Spain, Francisco Diaz-Gonzalez from UPC whose research is supported by the Ministerio de Economia, Industria y Competitividad, under the grant agreement number ENE2017-86493-R, Martin Kegel from CanmetEnergy who conducted this research in the framework of the Energy Innovation Program, and in consultation with Alaia Sola whose work is performed within the
GrowSmarter program (Horizon 2020 of the European Union under the grant agreement nr. 646456).

\section{References}

Akmal, M., Fox, B., Morrow, J.D., Littler, T. (2014). Impact of Heat Pump Load on Distribution Networks. IET Generation, Transmission \& Distribution 8,2065-2073.

Boneta, M.F., Sebi, C. (2012). The Challenges, Dynamics and Activities in the Building Sector and Its Energy Demand in Spain.

CIGRE. (2013). Benchmark Systems for Network Integration of Renewable and Distributed Energy Resources.

Dassault Systèmes. (2018). FMPy - Simulate Functional Mockup Units (FMUs) in Python. Retrieved from https://github.com/CATIA-Systems/FMPy/. Accessed April 17, 2018.

Dickert, J., Domagk, M., Schegner, P. (2013). Benchmark Low Voltage Distribution Networks Based on Cluster Analysis of Actual Grid Properties. IEEE Grenoble Conference PowerTech, POWERTECH 2013.

Eder, K., Widl, E., Gahler, C., Beigelbock, B., Judex, F. (2016). Co-Simulation for Control Design - A Case Study for Cross-Domain Collaboration. ASHRAE and IBPSA-USA SimBuild 2016.

European Commission. (2014). EU Building Database. Retrieved from https://ec.europa.eu/energy/en/eubuildings-database. Accessed May 21, 2018.

European Commission (EC). (2016). Proposal for a Directive of the European Parliament and of the Council Amending Directive 2010/31/EU on the Energy Performance of Buildings 2016/0381 (COD).

Flammini, M.G., Prettico, G. (2017). Interaction of Consumers, Photovoltaic Systems and Electric Vehicle Energy Demand in a Reference Network Model. International Conference of Electrical and Electronic Technologies for Automotive.

Fuentes, E., Waddicor, D.A., Salom, J. (2016). Improvements in the Characterization of the Efficiency Degradation of Water-to-Water Heat Pumps under Cyclic Conditions. Applied Energy 179, 778-789.

Jensen, S.Ø. et al. (2017). IEA EBC Annex 67 Energy Flexible Buildings. Energy and Buildings 155, 2534.

Maayan Tardif, J., Diaz-Gonzalez, F., Salom, J. (2018). Co-Simulation Platform for the Assessment of the Interaction Between Heat Pumps and the Low Voltage Grid at the Feeder Level. Universitat Politècnica de Catalunya - Barcelona Tech; Catalonia Institute for Energy Research - IREC

Marzal-Pomianowska, A., Diaz de Cerio Mendaza, I., Heiselberg, P., Bak-Jensen, B. (2016). Application of High-Resolution Domestic Electricity Load Profiles in Network Modelling. A Case Study of 
Low Voltage Grid in Denmark. CLIMA 2016 proceedings of the 12th REHVA World Congress.

Mateo, C. et al. (2018). European Representative Electricity Distribution Networks. International Journal of Electrical Power and Energy Systems 99,273-280.

Mehmedalic, J., Rasmussen, J. and Harbo, S. (2013). Grid Impact Studies of Electric Vehicles ; Reinforcement Costs in Low-Voltage Grids.

MODELISAR consortium. (2014). Functional Mock-up Interface for Model Exchange and Co-Simulation.

Müller, D. et al. (2015). Demand Side Management for City Districts. Building and Environment 91,283293.

Navarro-Espinosa, A., Mancarella, P. (2014). Probabilistic Modeling and Assessment of the Impact of Electric Heat Pumps on Low Voltage Distribution Networks. Applied Energy 127, 249266.

Ortiz, J., Fonseca i Casas, A., Salom, J., Garrido Soriano, N., Fonseca i Casas, P. (2016). Cost-Effective Analysis for Selecting Energy Efficiency Measures for Refurbishment of Residential Buildings in Catalonia. Energy and Buildings 128, 442-457.

Ortiz, J., Guarino, F., Salom, J., Corchero, C., Cellura, M. (2014). Stochastic Model for Electrical Loads in Mediterranean Residential Buildings: Validation and Applications. Energy and Buildings 80, 23-36.

Péan, T.Q., Salom, J., Ortiz, J. (2017). Potential and Optimization of a Price-Based Control Strategy for Improving Energy Flexibility in Mediterranean Buildings. Energy Procedia 122, 463-468.

Prettico, G., Gandale, F., Mengolini, A., Lucas, A., Fulli, G. (2016). Distribution System Operators Observatory. European Electricity Distribution Systems to Representative Distribution Networks.

Rodriguez-Calvo, A., Cossent, R., Frías, P. (2017). Integration of $\mathrm{PV}$ and $\mathrm{EVs}$ in Unbalanced Residential LV Networks and Implications for the Smart Grid and Advanced Metering Infrastructure Deployment. International Journal of Electrical Power and Energy Systems 91, 121-134.

Salom, J., Marszal, A.J., Widén, J., Candanedo, J., Byskov Lindberg, K. (2014). Analysis of Load Match and Grid Interaction Indicators in Net Zero Energy Buildings with Simulated and Monitored Data. Applied Energy 136, 119-131.

Salom, J., Widén, J., Candanedo, J., Byskov Lindberg, K. (2015). Analysis of Grid Interaction Indicators in Net Zero-Energy Buildings with Sub-Hourly Collected Data. Advances in Building Energy Research 9(1), 89-106.

Sartori, I., Ortiz, J., Salom, J., Ijaz Dar, U. (2014). Estimation of Load and Generation Peaks in Residential Neighbourhoods with BIPV: Bottomup Simulations vs. Velander. World Sustainable Building 2014 Conference, 17-24.
Schwalbe, R., Häusler, M., Stifter, M., Esterl, T. (2017). Market Driven vs. Grid Supporting Heat Pump Operation in Low Voltage Distribution Grids with High Heat Pump Penetration - an Austrian Case Study. 12th IEA Heat Pump Conference.

Solar Energy Laboratory Univ. of Wisconsin-Madison, TRANSSOLAR Energietechnik GmbH, CSTB Centre Scientifique et Technique du Bâtiment, and TESS - Thermal Energy Systems Specialist. (2017). Trnsys 18.

Tejero, A., Ortiz, J., Salom, J. (2018). Evaluation of Occupancy Impact In A Residential Multifamily NZEB Through A High Resolution Stochastic Model. 4th Building Simulation and Optimization Conference. Cambridge (UK).

Thurner, L., Scheidler, A. (2017). Pandapower Convenient Power System Modelling and Analysis Based on PYPOWER and Pandas. Fraunhofer IWES Universität Kassel

Toffanin, R. (2018). Energy Flexibility Strategies for Residential Buildings in Mediterranean Climates. Universitat Politècnica de Catalunya - Barcelona Tech; Catalonia Institute for Energy Research IREC.

Wang, K., Olaf Siebers, P., Robinson, D. (2017). Towards Generalized Co-Simulation of Urban Energy Systems. Procedia Engineering 198, 366-374.

Widl, E. et al. (2015). Simulation of Multi-Domain Energy Systems Based on the Functional Mock-up Interface Specification. Proceedings - 2015 International Symposium on Smart Electric Distribution Systems and Technologies, EDST 2015, 510-515.

Wilkening, H., Troyer, C. (2016). Modelling Residential Load Profiles and Its Applications.

Zangheri, P. et al. (2014). Heating and Cooling Energy Demand and Loads for Building Types in Different Countries of the EU. ENTRA NZE 